\title{
Spectral emissivity evaluation for materials used in microelectronics
}

\author{
by $\mathrm{H}$. Madura ${ }^{1}, \mathrm{H}$. Polakowski ${ }^{1}, \mathrm{~B}$. Więcek ${ }^{2}$ \\ ${ }^{1}$ Institute of Optoelectronics, Military Academy of Technology, 2 Kaliski str. 01-489 Warsaw, Poland, \\ ${ }^{2}$ Institute of Electronics, Technical University of Łódž, 18-22 Stefanowskiego str. 90-924 Łódž, Poland,
}

\begin{abstract}
This paper presents emisivity $(\varepsilon)$ measurements for some materials used in microelectronics, eg.: aluminium, silicon, germanium and diamond-like structures. In this work we emphasise emissivity evaluation for semitransparent and multilayer dielectric materials. The measurement was performed with the use of IR Spectrometer. For every sample we measured the reflected and transmitted energy, and evaluated material constants for various spectral ranges. The results of this work may be useful in calculating heat removal by radiation in microelectronic devices .
\end{abstract}

\section{Introduction}

Emissivity needs to be evaluated whenever we want to measure temperature with thermography. In order to simulate heat exchange by radiation we have to include emissivity as well. More accurate modelling requires spectral or temperature-dependent characteristics of emissivity [2]. It is quite difficult to get the correct value of the emissivity in practice because it depends upon many varying parameters, e.g.: temperature, wavelength, surface oxidisation, roughness, etc. Although there are known methods to measure the emissivity, it is still quite hard to get its precise value in general [2-4].

For opaque materials we can evaluate the directional emissivity as: $\varepsilon(\theta)=1-\rho(\theta)$, where $\rho(\theta)$ denotes the directional reflectivity ( $\theta$-angle). For metals, the emissivity strongly depends on their optical properties, especially the refractive index which is typically expressed as complex number $\left(n=n^{\prime}+i k^{\prime}\right)$.

The problem is much more difficult for semitransparent and multilayer structures which exist in microelectronics. To evaluate their emissivity we have to consider material transmission and internal reflections [1]. The electromagnetic wave propagation theory is applied for such cases, especially for dielectric layers with a thickness of the same order as the wavelength [1].

\section{Emissivity measurements}

The results of the reflection - transmission measurements and the calculated emission are presented in Fig.1-4. We measured both aluminium samples with different surface conditions and semiconductors, i.e. silicon and germanium covered by thin diamond-like layers. IR Spectrometer Model 1725 by Perkin-Elmer was used. All measurements were performed in a direction perpendicular to the surface of the investigated body in the spectral range of $1.5 \div 25$ $\mu \mathrm{m}$.

For opaque materials like aluminium we evaluate the spectral emissivity in the normal direction to the sample as

$$
\varepsilon_{n}(\lambda)=1-\rho_{n}(\lambda)
$$

where $\rho_{n}(\lambda)$ denotes the normal spectral reflectivity.

For semitransparent semiconductors or diamond-like samples we include transmission of the material as well. It is quite easy to evaluate emissivity for samples large in comparison with the wavelength, where there is no any internal reflections and wave interference effects. In such cases normal emissivity can be expressed as.

$$
\varepsilon_{n}(\lambda)=1-\rho_{n}(\lambda)-\tau_{n}(\lambda)
$$

where $\tau_{n}(\lambda)$ denotes the normal spectral transmissivity. 


\section{http://dx.doi.org/10.21611/qirt.1996.009}

For multilayer structures we include internal reflection, which obviously takes place in the practice (Fig.6). By off-line calculations using the optical constants identified during the measurement, one can find parts of the energy absorbed in every layer, what directly corresponds to the layer's emissivity.

Assuming that we have a partially transmitting layer (Fig.6.), the total absorption $A$ including the reflection from the second surface is [1]

$$
A=\frac{(1-\rho)(1-\tau)}{1-\rho \tau}
$$

Eqn. (3) was derived by net-radiation method, under the assumption of isothermal conditions (absorption does not increase the temperature). Similarly we can obtain the reflected and transmitted energy fractions, which were measured in this work.

$$
R=\rho \frac{1+(1-2 \rho) t^{2}}{1-\rho^{2} \tau^{2}} \quad T=\frac{\tau(1-\rho)^{2}}{1-\rho^{2} \tau^{2}}
$$

From eqn. (4) we can evaluate reflectivity $\rho$ and transmissivity $\tau$, as function of wavelength $\lambda$. Together with eqn. (2) and (3), these values can then be used to evaluate the emissivity for any dielectric material with and without internal reflections. Using eqn. (4) we assume material thickness much larger than the applied wavelength, otherwise we need to consider wave interference, as well. One could notice that $A=\varepsilon$ for large samples where there are no internal reflections and wave interference.

For multilayer semitransparent structures e.g.: silicon-diamond and germanium-diamond, more complex formulas are used (Fig.5) [1]:

$$
\begin{aligned}
& T=\frac{\left(1-\rho_{1}\right)\left(1-\rho_{2}\right)}{1-\rho_{1} \rho_{2} \tau^{2}} \\
& A=\frac{\left(1-\rho_{1}\right)\left(1+\rho_{2} \tau\right)(1-\tau)}{1-\rho_{1} \rho_{2} \tau^{2}} \\
& R=\frac{\rho_{1}+\left(1-2 \rho_{1}\right) \rho_{2} \tau^{2}}{1-\rho_{1} \rho_{2} \tau^{2}}
\end{aligned}
$$

where $\rho_{1}, \rho_{2}$ are the reflectivities for both layers and $\tau$ the transmissivity for the upper one.

For both the single and the multi-layer structure we measure $R(\lambda)$ and $T(\lambda)$, as shown in Fig.1-4. Using eqn. (4) and the relation $\rho=\left(n-n_{a}\right)^{2} /\left(n+n_{a}\right)^{2}$, where $n_{a}$ is the reflective index for air (typically=1), we yield $n(\lambda)$. In a more precise calculation we even can provide $n_{a}(\lambda)$ [5], as Cauchy or Sellmeier formulas:

$$
n_{a}=1+\frac{0.00057387 \lambda^{2}}{\lambda^{2}-595260} \quad \text { where } \lambda[\stackrel{\prime \prime}{A}]
$$

In Table 1 we present radiation properties for silicon and germanium. $R, T, A$ denote the fraction of the reflected, transmitted and absorbed energy. $A$ corresponds to the emissivity of the semitransparent window if we take into consideration internal reflection. However, using (4) we can find the reflectivity $\rho$ the transmissivity $\tau$ and the emissivity $\varepsilon$ of the thick material when there are no internal reflections. 
Table1. Radiation properties for semiconductors

\begin{tabular}{|l|c|c|c|c|c|c|c|}
\cline { 2 - 7 } \multicolumn{1}{c|}{} & $\mathrm{R}$ & $\mathrm{T}$ & $\mathrm{A}$ & $\rho$ & $\tau$ & $\varepsilon$ & $\mathrm{n}$ \\
\hline $\begin{array}{l}\mathrm{Si} \\
2.5-5.5 \mu \mathrm{m} .\end{array}$ & 0.46 & 0.53 & 0.01 & 0.30 & 0.29 & 0.39 & 3.43 \\
\hline $\begin{array}{l}\mathrm{Si} \\
8-12 \mu \mathrm{m} .\end{array}$ & 0.38 & 0.36 & 0.26 & 0.30 & 0.21 & 0.48 & 3.45 \\
\hline $\begin{array}{l}\mathrm{Ge} \\
2.5-5.5 \mu \mathrm{m} .\end{array}$ & 0.54 & 0.46 & 0.00 & 0.37 & 0.37 & 0.26 & 4.10 \\
\hline $\begin{array}{l}\mathrm{Ge} \\
8-12 \mu \mathrm{m} .\end{array}$ & 0.54 & 0.45 & 0.01 & 0.37 & 0.37 & 0.26 & 4.14 \\
\hline
\end{tabular}

The parameter values obtained agree with published values and measured by other methods, e.g. the refraction indexes from [5] are: $n_{\mathrm{Ge}}=3.99, n_{S i}=3.49$. For semiconductor multilayers we only provide the total amount of energy absorbed in whole structure. More detailed investigations need to include the incident and reflective wave interference, because the diamond tickness is of the same order as the infra-red wavelength.

Table 2. Radiation proprieties for semiconductor multilayers

\begin{tabular}{|c|c|c|c|}
\cline { 2 - 4 } \multicolumn{1}{c|}{} & $\mathrm{R}$ & $\mathrm{T}$ & $\mathrm{A}$ \\
\hline $\mathrm{Si}+\mathrm{C}$ & 0.40 & 0.54 & 0.06 \\
\hline $2.5-5.5 \mu \mathrm{m}$. & & & 0.30 \\
\hline $\mathrm{Si}+\mathrm{C}$ & 0.38 & 0.32 & 0.03 \\
\hline $\begin{array}{c}\mathrm{Ge}+\mathrm{C} \\
2.5-5.5 \mu \mathrm{m} .\end{array}$ & 0.43 & 0.54 & 0.06 \\
\hline Ge+C & & & \\
$8-12 \mu \mathrm{m}$. & 0.50 & 0.44 & \\
\hline
\end{tabular}

For aluminium we provide the polynomial approximations of the emissivity over the spectral range used by thermography i.e. $2.5-14 \mu \mathrm{m}$ as

$$
\varepsilon(\lambda)[\%]=c_{0}+c_{1} \lambda^{1}+c_{2} \lambda^{2}+c_{3} \lambda^{3}+c_{4} \lambda^{4}+c_{5} \lambda^{5} \quad \lambda[\mu m]
$$

Table 3. Polynomial approximation of emissivity for aluminium

\begin{tabular}{|l|c|c|c|c|c|c|}
\cline { 2 - 7 } \multicolumn{1}{c|}{} & $\mathrm{c}_{0}$ & $\mathrm{c}_{1}$ & $\mathrm{c}_{2}$ & $\mathrm{c}_{3}$ & $\mathrm{c}_{4}$ & $\mathrm{c}_{5}$ \\
\hline Aluminium & 16.87 & -5.64 & 5.22 & -1.37 & 0.13 & -0.004 \\
\hline $\begin{array}{l}\text { Anodised } \\
\text { aluminium } \\
\text { (black) }\end{array}$ & -31.36 & 54.88 & -2.04 & -1.65 & 0.22 & -0.008 \\
\hline $\begin{array}{l}\text { Anodised } \\
\text { aluminium } \\
\text { (non-black) }\end{array}$ & 22.75 & 20.04 & 4.77 & -2.13 & 0.22 & -0.007 \\
\hline $\begin{array}{l}\text { Aluminium } \\
\text { +diamond }\end{array}$ & -37.61 & 63.45 & -1.23 & -4.63 & 0.19 & -0.007 \\
\hline
\end{tabular}

\section{Conclusions}

In this paper we present measurements of the spectral emissivity for semiconductors and aluminium with different surface coatings. For semiconductors the emissivity is quite low, except for silicon in the long-wavelength range. It is due to local minima of the reflectivity and the transmissivity for 8,10 and $12 \lambda_{0} ;$ where $\lambda_{0}=1.13 \mu \mathrm{m}$. This corresponds to the energy gap in silicon $(\sim 1.1 \mathrm{eV})$. This effect was not observed for germanium. A thin layer of diamond on the 
semiconductor does not enhance the emissivity very much, but works as antireflective coating, especially in the short wavelength range. For aluminium widely used in microelectronics both the anodising and the covering by the diamond increases the emissivity significantly. The measurement performed for semiconductors allow to identify the wavelength-dependent optical parameters for infra-red, e.g. $n, \rho, \tau$.

\section{REFERENCES}

[1] Siegel (R.) and Howell (J).- Thermal radiation heat transfer. New York, Hemisphere Publishing Corp, 1989, p.721-750

[2] Burakowski (T.), Giziński (J.) and Sala (A.). - Promienniki podczerwieni, Warsaw, WNT, 1970, p. 65-80. [in Polish].

[3] Sala (A.) - Radiacyjna wymiana ciepla. Warsaw, WNT, 1982 [in Polish]

[4] Więcek (B.), Pacholiki (J.), Technical Method of Emmisivity Correction in Thermography, Quantitative Infrared Thermography QIRT'94, August 23-26 1993, Sorento, Italy, p. 124-130

[5] Lide (D.). Handbook of chemistry and physics. $71^{\text {st }}$ Edition, 1990-1991, CRC Press, USA 1991, ISBN-0-8493-0471-7

Aliminium (non-polished)

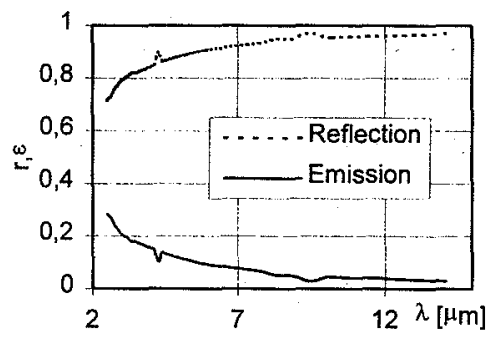

Anodised aluminium (black)

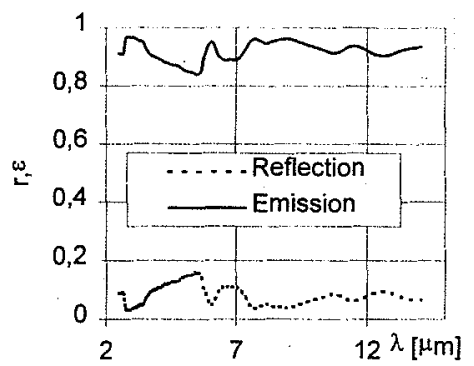

Aluminium + diamond

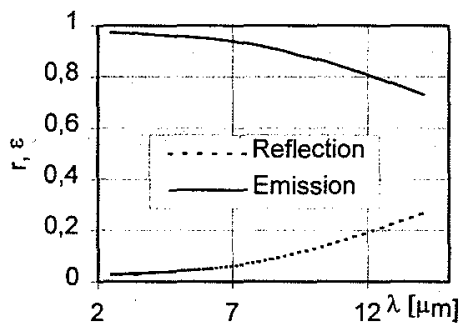

Anodised alumunium (non-black)

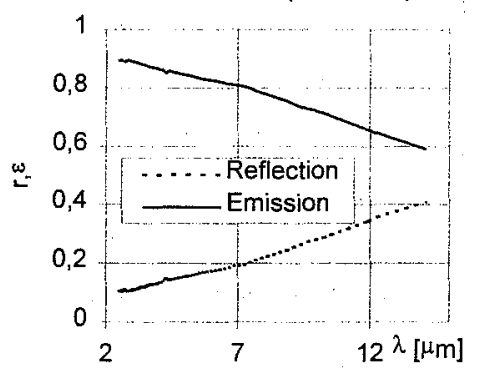

Fig.1. Reflectivity and emissivity for aluminium in the range $2.5-14 \mu \mathrm{m}$. 

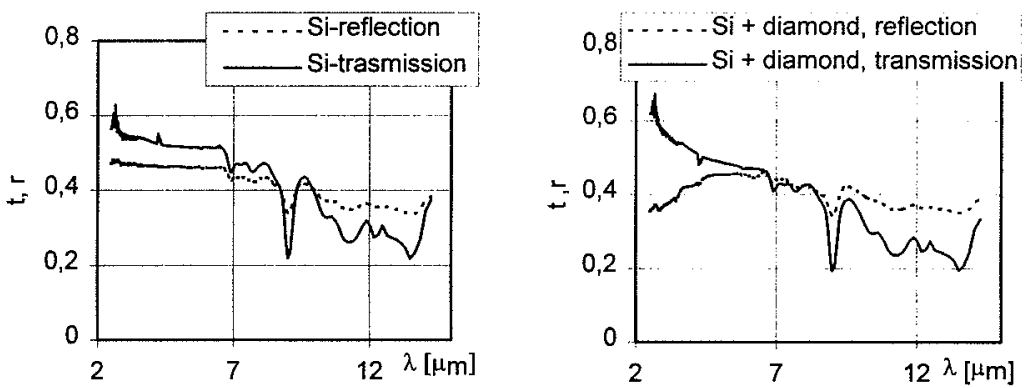

Fig.2. Reflectivity and transmissivity for silicon the range $2.5-14 \mu \mathrm{m}$.
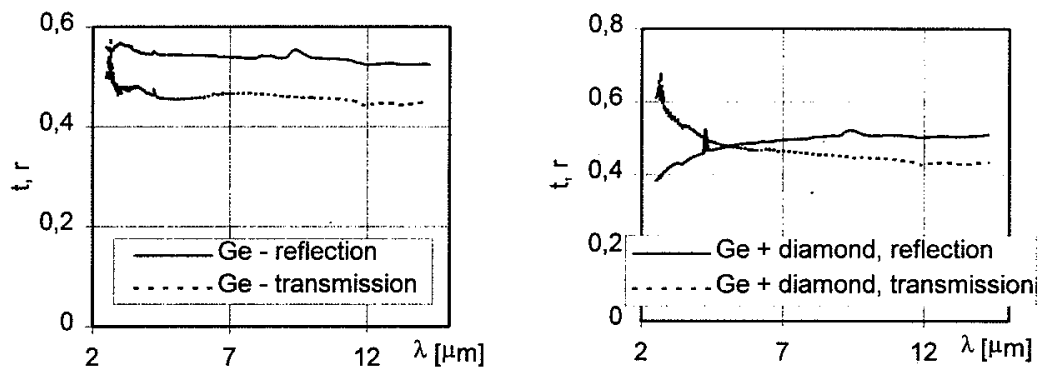

Fig.3. Reflectivity and transmissivity for germanium the range $2.5-14 \mu \mathrm{m}$
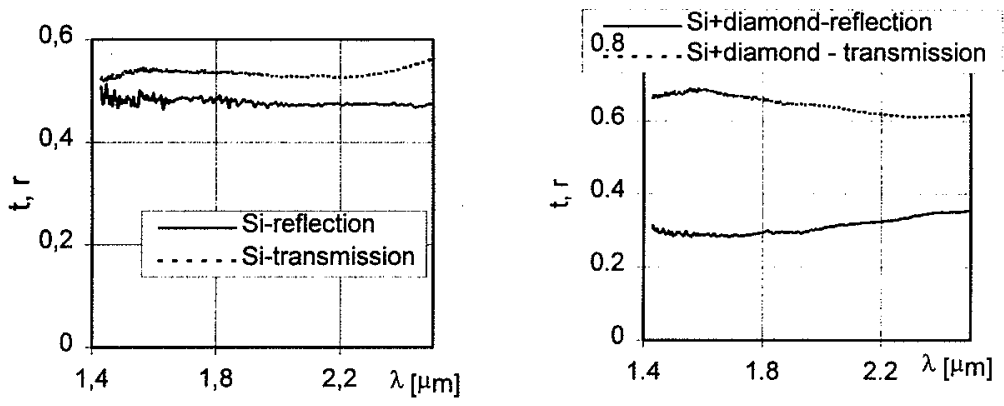

Fig.4. Reflectivity and transmissivity for silicon in the range 1,4-2.5 $\mu \mathrm{m}$ 

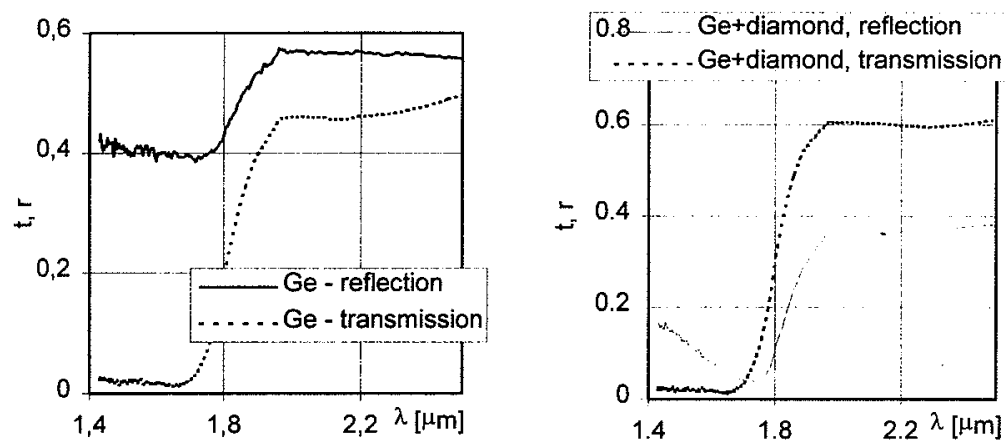

Fig.5. Reflectivity and transmissivity for germanium the range 1,4-2.5 $\mu \mathrm{m}$
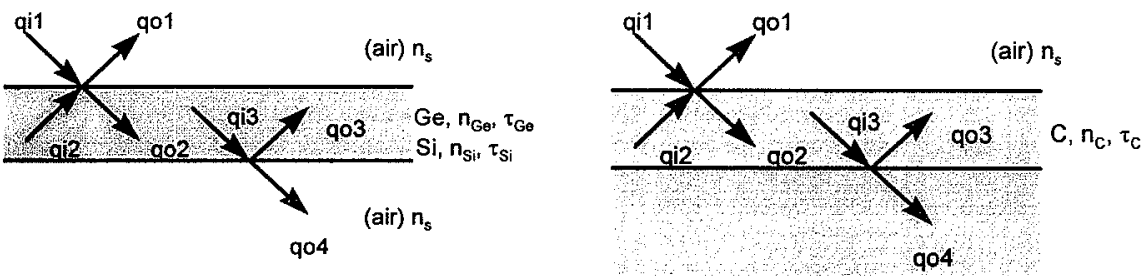

$$
\mathrm{Si}, \mathrm{r}_{\mathrm{si}} \mathrm{r}_{\mathrm{s}}
$$

Fig. 6. Semitransparent and multilayer semiconductor structures for emissvity evaluation 